\title{
2 What Constitutes an Effective Language MO0C?
}

\begin{abstract}
Language MOOCs are in their early stage of development. Given this, we have an opportunity to take from the best of current language learning theory and abandon both ineffective methodologies of the past, as well as methodologies of other fields. This is best accomplished by adopting the ideologies of cMOOCs, while maintaining some of the structure of xMOOCs needed to help students navigate complex technologies and information in languages that are not their own. Maximizing engagement, community membership, communication, and creativity will ensure that Language MOOCs, as we move forward, will offer exciting and engaging ways to interact with language as subject matter.
\end{abstract}

Keywords: MOOC, xMOOC, cMOOC, second language learning, motivation, engagement, assessment

\subsection{Introduction}

"All growth is a leap in the dark, a spontaneous unpremeditated act without the benefit of experience." - Henry Miller

If MOOCs are in their infancy (cf. Marques, 2013), Language MOOCs are positively neonatal. Presently, only a handful of such MOOCs exist. Two of the largest purveyors of MOOCs, edX and Coursera, do not include 'languages' as a category in their directory of courses, and searching both (using communication or humanities as search keywords) reveals only a few such courses, all focused on English writing. Instreamia.com, a newer newcomer in this land of newcomers, bills itself as "the Future of Language Learning." It launched in 2012, and currently offers three levels of Spanish for English speakers, and courses in business and academic English for Spanish and Portuguese speakers. Various sites offer materials in language learning, but they do not qualify as MOOCs, failing to incorporate one or more of the main concepts of a MOOC:

Massive: enrollment is large, in the thousands, even tens of thousands of students. Open: enrollment is free and not restricted to students by age or geographic location. Online: the course takes place entirely online, with no required face-to-face (f2f) component. Course: the materials comprise a course, usually with a syllabus and schedule, with the guidance or presence of an instructor and/or instructional assistance. 
In fact, the promise of Language MOOCs seems to be downplayed in the literature, if not dismissed outright. In some cases, the reasons for this dismissal are vague, as in Stevens (2013):

[F]or language learning, a MOOC would not be a good means of teaching grammatical structures, unless the students were tasked with learning grammar inferentially and from one another. In some theories of language learning, they would learn through being motivated to communicate with one another and through providing one another with linguistic data that they would be constantly processing. (p. 9)

It is unclear why, in this case, grammatical structures would be impervious to instruction, while concepts in other fields, such as programming language, biology, or economics escape such judgment. The underlying assumption is that inferential learning is the only way to teach language.

Other arguments seem to rely on faulty premises, as in the discussion by Romeo (2012) as to why MOOCs are not appropriate in the English language-learning environment:

ESL [English as a Second Language] is all about exactly what the MOOCs specifically, and selfstudy in general, cannot [sic] do. The whole reason that anyone even takes an ESL course is not out of curiosity, or general interest, it is because they have to.

The premise that no one takes an English course out of curiosity or interest is so clearly fallacious that it requires no rebuttal. Furthermore, even if it were true, there is ample evidence that extrinsic motivation is a legitimate reason for language learning that does not negate the idea of learner autonomy (cf. Schmidt, Boraie, \& Kassabgy, 1996; Dörnyei, 2001).

It is, however, because of the newness of Language MOOCs that we are presented with the opportunity to get it right-to capture the best of what we know about language learning as well as the best of what we know about online educational experiences, especially the successes and failures of previous MOOCs. We can, and should, allay the fears that MOOCs are merely a return to teacher-fronted, "drill and kill" language instruction that cannot, by its very medium, be successful at the task of teaching a language.

\section{2 cMOOCs, xMOOCs}

"First we build the tools, then they build us." - Marshall McLuhan

In their short lifespan, MOOCs no longer comprise a singular concept. They are already categorized, forming a new educational typology. Most notable is the bifurcation between cMOOCs, the original conception of a MOOC (Downes, 2008; 2013) and 
xMOOCs. Connectivist MOOCs (the 'c' in cMOOC) were seen as distributed networks "in the sense that they do not run on a single website or with a centralized core of content; the content in cMOOCs is networked. Participants are encouraged to meet in locations of their choosing and organize themselves" (Mackness, 2013). In contrast, xMOOCs (the ' $x$ ' comes from the open course model originally formed as MITx, which was then joined by other universities, and has evolved into edX.org) are centralized networks "convened on a designated platform; ... the course runs principally on the main platform, where interaction takes place in discussion forums" (Mackness, 2013).

The advantages the $\mathrm{CMOOC}$ format is its emphasis on interaction and community building. In language learning, this seems to coincide neatly with the goals of most classroom pedagogy, especially that of the communicative language teaching (CLT) approach. This approach is characterized briefly as:

\footnotetext{
[A] movement away from traditional lesson formats where the focus was on mastery of different items of grammar and practice was through controlled activities such as memorization of dialogs and drills, and toward the use of pair work activities, role plays, group work activities and project work. (Richards, 2006, p. 4)
}

The communicative approach is seen as one in which the focus is on the learner's ability to use and adapt language in authentic situations. The cMOOC format fits well with the goals of CLT.

The disadvantages of the cMOOC format are that it is often described as difficult to navigate, "overwhelming" and sometimes relying on materials that are inaccessible in some geographic locations (Jordan, 2013). Brennan (2013) states:

Cognitive load, and Prior Knowledge are key here, at times ignored by Connectivism, and either dismissed or not designed for in both theory and practice. They have a huge determining effect on what we can learn, and how we learn it, and are particular issues for novice learners.

The added difficulty for language learning is that in a truly communicative MOOC, students are using the medium of instruction as the medium of communication. This increases the cognitive load in a different way than a complex content-area course in astronomy, for example, might. Imagine taking a computer programming course where every exchange had to be written in computer code-no 'authentic' language allowed. Layer that in a true cMOOC format, asking learners of a language to navigate a complex web written in the target language, and the overwhelming nature of the task multiplies.

xMOOCs, on the other hand, provide what might be seen as a more "familiar" structure for learning. An instructor guides the course, often through a syllabus and a sequence of activities. xMOOCs are most often sponsored by universities, and thus, in some ways are seen to mimic the structure of university courses. It's unfortunate that rather than a dichotomy between xMOOCs and cMOOCs, there has developed a kind of hierarchy, where many consider cMOOCs to be superior in form and function. For 
example, Siemens (2012) states, "cMOOCs focus on knowledge creation and generation whereas xMOOCs focus on knowledge duplication". There is a danger, however, in xMOOCs replicating the worst of what exists about online learning-rote drills, decontextualized language, inauthentic activities, intrusive interface, etc. As Caulfield (2013) states, "[I]f xMOOCs want to truly have persistent, effective communities they are going to have to build the community not around success in the course, but around larger, more authentic applications of course content”.

\subsubsection{On not Taking a Cue from CALL}

Computer Assisted Language Learning (CALL), of course, has a much longer history than do MOOCs. CALL was originally conceived of as "the search for and study of applications of the computer in language teaching and learning” (Levy, 1997, p. 1). Early experiments with CALL date back to the days of mainframe computers. However, from its inception, it was often associated with non-communicative activities, largely because of the limitations of networks until recent years. There were other limitations, as Jarvis and Krashen (2014: 1) state in a recent article:

When the term CALL (Computer-Assisted Language Learning) was introduced in the 1960s, the language education profession knew only about language learning, not language acquisition, and we assumed the computer's primary contribution to Second Language Acquisition (SLA) were programs based on traditional language learning.

Much of early CALL centered around locally-installed software programs that offered primarily activities in which the learner consumed language, but did not produce it, typically: grammar drills, listening activities, or reading comprehension tasks. When the Internet became more widely used on home computers, rather than in classrooms or workplaces, web-based activities appeared. However, these often mimicked the activities that were found in the local software programs. One of the most popular early English language learning websites was Dave's ESL Café (http://www.eslcafe. $\mathrm{com} /$ ), which still exists, and is still popular (though one might argue more as a job site than for language learning. Such is the way of the today's world.). The language activities there still imitate the "grammar workbook" style of learning English, with grammar lessons followed by quizzes of the following style (see Figure 2.1): 


\section{Prepositions1 Quiz}

\section{Special Instructions:}

After answering "all" the questions, press the 'submit' button, and your answers will be automatically checked in seconds. Have fun while you leam!

1. Dave graduated

Pepperdine University.

for

from

at

2. I'm not familiar

with

of

to

3. The jar is filled candy.

by

of

with

4. The book is

in

on

to

California.

Figure 2.1: Preposition quiz (Source: http://www.eslcafe.com/quiz/prep1.html)

In fact, it was not until the widespread implementation of Web 2.0 technologies, or more specifically, social networking sites like Facebook and Twitter, in addition with a vast availability of online resources, that the possibility of online language learning that involved meaningful communication was even possible.

\subsubsection{The Opportunity for an LMOOC}

Given the strengths and weaknesses of both cMOOCs and xMOOCs, as well as the nature of language learning that makes it different from learning physics or statistics, for example, it may be time to add another node to the MOOC taxonomy: that of the LMOOC, or Language MOOC. The LMOOC, in imitating the best practices of language teaching and learning, is an eclectic mix of practices and tools aiming to engage students in the use of the target language in meaningful and authentic ways. The next section addresses the characteristics of an ideal LMOOC.

\subsection{Making the Massive Human(e)}

\footnotetext{
"In the future there will be so much going on that no one will be able to keep track of it." - David Byrne
} 
About the MOOC I designed and teach, Principles of Written English (College Writing 2x, BerkeleyX, averaging approximately 50,000 participants per five-week segment of the course), a student recently tweeted (see Figure 2.2):

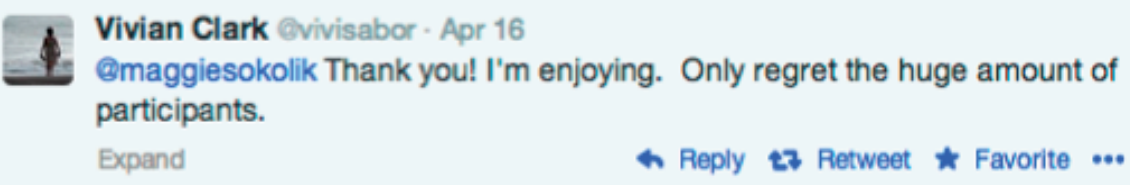

Figure 2.2: Student tweet about course enrollment

Participants are often surprised, in fact, to find out the large number of enrollments in a MOOC, whether it's an XMOOC or a cMOOC. I was similarly surprised. I had feared that as many as 10,000 would ultimately enroll; my fear was amplified when I saw that 10,000 enrolled the day that registration opened. One of the goals in a Language MOOC has to be to make that number manageable, even irrelevant, to the task of learning.

The proposal here for a model for an LMOOC comes largely from this experience in developing, designing, and teaching a MOOC for English language learners. It comes from some of its successes, but mostly from its many lessons learned. Some of the suggestions pertain to platform features that should be developed if LMOOCs are going to be implemented successfully, leveraging the techniques of language teaching and learning we know to be successful.

\subsubsection{Engagement and Interaction}

As mentioned, one of the cornerstones of communicative language teaching, as well as a feature of cMOOCs is a high level of engagement and interaction. In Language MOOCs, it is important that the engagement and interaction take the form of authentic communication, primarily among students. While self-check activities, such as quizzes or short-text entry can be used for students to confirm their understanding of basic concepts, most would agree that the goal of language learning is " $[\mathrm{k}]$ nowing how to maintain communication despite having limitations in one's language knowledge" (Richards, 2006, p. 3).

In xMOOCs of today, communication takes place primarily through threaded discussion platforms. Unfortunately, these platforms often fall short in their ease of use and performance. As McGuire (2013: 31) states:

Most MOOC discussion forums have dozens of indistinguishable threads and offer no way to link between related topics or to other discussions outside the platform. Often, they can't easily be sorted by topic, keyword, or author. As a result, conversations have little chance of picking up steam, and community is more often stifled than encouraged. 
cMOOCs, on the other hand, tend to leverage existing social media sites or find ways of connecting for discussion. Mackness (2013) states, " Participants are encouraged to meet in locations of their choosing and organise themselves".

The different ways of organizing discussion between cMOOCs and xMOOCs is a critical one, and I would argue that neither is perfect. The architectural issues with centralized discussion areas are a legitimate concern. Relying on current social media is also concerning. Using Facebook or Twitter, for example, leaves out students in certain geographic areas due to government restrictions, or leaves out students with concerns about privacy issues with those platforms.

However, discussion is the heart of a Language MOOC. The most important work goes on there, whether it's discussing a reading, sharing writing, or testing out a grammar point. What should discussion look like in an LMOOC?

\subsubsection{Student Self-Organization in LMOOCs}

One trend I noticed in College Writing 2x was for students to self-organize in discussion groups outside of the edX.org platform. In other words, there was a natural trend towards something that looked more like a cMOOC approach to discussion. However, their reliance on established social media platforms (primarily Facebook) meant that only a fraction of students could participate there.

\subsubsection{Instructor Presence}

Another issue that has arisen is the desire for the instructor to be more visible in discussions. In a study of instructor involvement in a MOOC, Tomkin and Charlevoix write of a study they did of one MOOC:

The active involvement of the professor did not matter in this MOOC. This course was carefully constructed, and the participants were highly-educated and motivated by a strong interest in the material. As it is likely that the students in many MOOCs fit this description, it is reasonable to suppose that this result holds generally for MOOCs offered today. (2014, p. 76)

I would argue that for LMOOCs, however, instructor presence is important (though perhaps not necessary) to help build community. Kop (2011) explains:

[T] here are some other conditions that clearly encouraged people's involvement and engagement in learning in a connectivist learning environment, including the "social presence" of the facilitators and of participants, which enhanced the "community" forming and the sense of belonging that built confidence and stimulated active participation. 
However, given the structure of both social media and centralized discussion areas of xMOOCs, the presence of the instructor or of designated facilitators may go unnoticed.

\subsubsection{An LMOOC Structure for Engagement}

Because LMOOCs rely heavily on discussion as part of the learning, a different way to facilitate online discussion should be built. This platform should allow for selforganization, by allowing users to create user groups and follow selected colleagues' posts (think of Facebook friend lists or Twitter followers). As learning another language entails more than learning to write in that language, it should allow for voice/ video posts as well. In order to build stronger bonds, and create a sense of instructor presence, this platform should allow for users to see when instructors and facilitators are online and available for contact. Kop points out: "[T]he closer the ties between the people involved, the higher the level of presence and the higher the level of engagement in the learning activity” (Kop, 2011).

\subsubsection{Talking Heads - Good Music, Bad Video}

Instructional style in most xMOOCs rely on video lectures, typically short ones. I admit when I was first approached about teaching a MOOC, my first resistance was in creating video lectures. (I do not lecture in my face-to-face classes, so why would I lecture online?) Apart from a few notable TED videos, my own attention for online lectures is short. I could not imagine MOOC participants' attention being any different. Guo, Kim, and Rubin (2014) state it succinctly:

[T]o maximize student engagement, instructors must plan their lessons specifically for an online video format. Presentation styles that have worked well for centuries in traditional in-person lectures do not necessarily make for effective online educational videos.

It is clear that almost no one can learn a language well by listening to others explaining it. LMOOC videos can be engaging, but only by leveraging the idea of realia, objects and material from everyday life used as teaching aids, or even better, virtual realia, "digitized objects and items from the target culture which are brought into the classroom as examples or aids and used to stimulate spoken or written language production" (Smith, 1997). 


\subsubsection{LMOOC Approach for Video Engagement}

LMOOCs should expand the idea of what video can be. There are many models of excellent use of video in language learning, from the U.S. public television's "French in Action" (WGBH, 1987), a story-based approach to authentic language learning, to Michael Marzio's person-on-the-street-style video-clips and lessons on http://www. real-english.com/. LMOOC instructors and producers should avoid talking head videos, and immerse the participant in a rich environment that will encourage reflection and discussion.

\subsubsection{Forms of Assessment}

In an educational environment that is often assessment-driven, it is not surprising that xMOOCs, whose model can be seen as the more traditional approach, incorporate quizzes and other types of assessment into the architecture. cMOOCs adopt a different tactic, as described by Crowley:

In xMOOCs, learners complete assessments (quizzes or peer-reviewed assignments) that evaluate their comprehension of a topic as it is understood from the instructor's view. In cMOOCs, learners share their insights as they go through the knowledge-building process (e.g., via status updates or blog posts) and self-assess their learning paths. (2013, p. 2)

The question of what needs to be assessed, how often, and by whom remains an item of debate in LMOOCs. However, given that some instructors, as well as students, want or expect assessment, what kinds of assessment make sense in an LMOOC?

For MOOCs that utilize open-ended responses, such as essays or short-answer quizzes, or spoken/video responses, assessment is difficult. There are three models for assessment that are commonly used: self-assessment, peer-assessment, and machine-assessment (for written work). The third of these is in its infancy, and the results are questionable (cf. Deane, 2013), so it will not be considered here.

\subsubsection{Peer-Assessment}

Peer grading often entails training participants, using a rubric developed by the instructor, to read each other's work and assess it on a number of points. Given the large number of students in a MOOC, this is often seen as the only viable method:

As MOOCs become more widespread, the need for reliable grading and feedback for open ended assignments becomes ever more critical. The most scalable solution that has been shown to be effective is peer grading. (Piech et al., 2013). 
However, peer grading comes with an assortment of issues. Even with training, some students are not prepared or willing to provide useful feedback to others. Many students see peer-feedback as unhelpful, and sometimes hurtful. In language learning, the different levels of proficiency may also affect a participant's ability to assess language that is at a higher level of proficiency. Finally, there is also evidence that national bias exists when peer grading, with students assessing work of peers from their own countries higher than work from other countries (Kulkarni, et al., 2013).

\subsubsection{LMOOCs and Assessment}

While many are working at improving the peer-assessment processes (cf. Piech et al., 2013), structured self-assessment may provide a better path in an LMOOC. A self-assessment instrument can be created by a course designer alone, or in tandem with beta-testers or participants. (An example self-assessment rubric is found in the Appendix.) Kulkarni et al., citing the work of several researchers, explain that selfassessment "helps students reflect on gaps in their understanding, making them more resourceful, confident, and higher achievers...and provides learning gains not seen with external evaluation" (2013, p. 3).

On the other hand, informal peer-feedback, that is, seeking feedback through discussion, can offer more meaningful commentary, since it is voluntary, and not part of a larger, mandatory assessment scheme. In a voluntary system, students choose which items to give feedback one, presumably based on their interest and level of expertise they feel in giving feedback.

If assessment is to be used in an LMOOC, a system that offers a combination of auto-scored multiple-choice or text-input items, in tandem with self-evaluation, and an effective discussion mechanism (see section above) may be the most satisfying for participants.

\subsubsection{My MOOC is Bigger than your MOOC}

In the face-to-face world of education, few of us would ever be boast about having large classes. In fact, it is usually with satisfaction that we are assigned the small seminar, the limited enrollment writing class, the 15-person language course. But the acronym MOOC begins with massive, and there is a degree of success that is associated with large enrollments. One criticism of MOOCs on the most prominent platforms has been that they are driven by superstar professors (Glader, 2012) striving for huge enrollments. 


\subsubsection{Conflating Numbers with Success}

When I was beginning my MOOC, I was asked by one of its co-sponsors, the US Department of State, how I would define success. Normally in a face-to-face course, success is defined externally by the university as achieving certain passing marks, or by me as the instructor, as feeling that students have understood most of the course material in a meaningful way, or by the students themselves, feeling that they have learned something of use to them in their future.

However, there is a tendency to equate popularity with success. Kulkarni et al., warn against the dangers of confusing high enrollment numbers with "success":

Typing one's email address into a webpage is not the same as showing up for the first day of a registrar enrolled class. It's more like peeking through the window, and what the large number of signups tell us is that lots of people are curious. How can we convert this curiosity into meaningful learning opportunities for more students? (2013, p. 25)

For a MOOC, given the large numbers - however real they may be - success has to be driven by students themselves. As no student is compelled to sign up, attend, or even participate in any way in a MOOC, our usual ways of measuring success have to be questioned.

\subsubsection{There are No Dropouts Here}

A related concern for MOOCs has been the so-called dropout rates, as reported here:

Coursera, and other MOOC providers like Edx, have been seeing massive drop-out rates to go along with their massive enrollment. The New York Times reported that of the 154,763 students who registered for MITx's circuits and electronics course only 7,157, or 4.6\%, passed and less than $50 \%$ of registered students made it to the first problem set. (Wukman, 2012)

However, given the nature of enrollments as stated previously, and the fact that there is nothing to compel anyone to start, continue, or complete a MOOC, many have started to question the concept of 'drop-out' in a MOOC. As Devlin (2013) writes, “[A] pplying the traditional metrics of higher education to MOOCs is entirely misleading. MOOCs are a very different kind of educational package, and they need different metrics - metrics that we do not yet know how to construct”.

\subsubsection{Defining Success in an LMOOC}

As Devlin (2013) also points out, "MOOCs mean so many different things to so many different people, only time will tell which sections of society they most serve." We know this to be the case for language learning as well. Students may decide to learn a language in order to achieve minimal competence for an upcoming holiday, or to 
communicate with friends and relatives in another language, or to learn it well enough to read works of literature, or to pursue a degree in higher education. In answering the question about how I will define success in my MOOC, I have to defer the questions to the participants themselves. Given that students join MOOCs for very different reasons, definitions of success will vary accordingly. For the student who has external motivation, for example, learning specific vocabulary or structures to communicate while traveling, success will be measured in achieving those goals. For students who wish to pursue higher education, success may be dependent on goals that are more than language learning itself; they may also involve the learning of proper tone or style for academic discourse.

\subsection{Conclusion}

“MOOCs, regardless of underlying ideology, are essentially a platform” - George Siemens, 2012

In examining the differences between xMOOCs and cMOOCs, the idea that Language MOOCs might require a platform with characteristics that are particular to the subject matter has emerged. This platform needs a richer, more nuanced set of communication tools suitable for the task of language learning; use of instructional videos that present examples of the language and culture of study without resorting merely to expository lectures on linguistic structures; and assessment tools that are sensitive to the range of abilities and goals of students.

Until such a platform emerges for language instruction, those of us who design and teach LMOOCs will need to look at the tools available and determine how best to leverage them to meet our goals. The organization of these MOOCs may look like cMOOCs, or like xMOOCs, but in the end the platform may be less important than the goals and planning of those who design the MOOCs themselves.

Given that LMOOCs are in the first few days of their infancy, it is critical to pause and consider carefully the learning experience we want to offer to participants. As language educators, we are aware of the complex task that teaching and learning a language entail. If we merely transfer the type of instruction found in any languagelearning textbook, or in the early attempts at CALL, to the MOOC platform, we cannot be said to be transforming language instruction-instead, we are merely retyping it.

\subsubsection{Summary: Tips for a Successful LMOOC}

To summarize the points made in this chapter, instructors and designers who are developing LMOOCs should consider the following suggestions:

1. Maximize engagement and interaction 
Consider the ways in which students can participate on the MOOC platform. Students will be more engaged in using language if they can communicate among themselves. The focus of learning should be in interaction, and not just in absorbing course materials.

2. Facilitate, but do not manage, self-organized learning

Suggest ways that students might self-organize through social media or face-toface meetings when possible, but do not manage these groups. Students will, in fact, most likely organize these groups without instructor participation at all.

3. Create an instructor presence

Students need to know that there is a human instructor participating in the LMOOC. Take time to comment on student discussions in the MOOC, make regular announcements, create a Facebook page or Twitter feed to support the class, etc.

4. Use video for engagement

Think about video as a source of authentic language materials, not just as a way to deliver a lecture. Use video as an opportunity for students to become engaged in culture as well as the language itself.

5. Define success

As you develop a Language MOOC, think about what success will mean in the course. Encourage students to think of their own goals, and how they will use the course to achieve them.

6. Match the goals of assessment with its form

As you think about the goals of the course, consider what type of assessment matches those goals. If the course focus is on writing, for example, then assessment types should evaluate writing in a meaningful way, such as through peer and self-assessment, rather than through multiple-choice questions.

\section{Bibliography and Webliography}

Brennan, K. (2013). In Connectivism, no one can hear you scream: A guide to understanding the MOOC novice. Retrieved April 9, 2014, from the Hybrid Pedagogy Web site: http://www. hybridpedagogy.com/Journal/in-connectivism-no-one-can-hear-you-scream-a-guide-to-understanding-the-mooc-novice/

Byrne, D. (2007). In the future. The knee plays. New York: Nonesuch Records.

Capretz, P. (1987). French in action. Boston, MA: WGBH Public Television.

Caulfield, M. (2013). xMOOC communities should learn from CMOOCs. Retrieved April 1, 2014, from the Educause Web site: http://www.educause.edu/Blogs/Mcaulfield/Xmooc-CommunitiesShould-Learn-Cmoocs

Crowley, J. (2013). cMOOCs: Putting collaboration first. Campus Technology. Retrieved March 19, 2014 from the Campus Technology Web site: http://campustechnology.com/ Articles/2013/08/15/cMOOCs-Putting-Collaboration-First.aspx

Daniel, J. (2012). Making sense of MOOCs: Musings in a maze of myth, paradox and possibility. Retrieved March 9, 2014, from the Journal of Interactive Media in Education Web site: http://jime.open.ac.uk/2012/18

Deane, P. (2013). On the relationship between automated essay scoring and modern views of the writing construct. Assessing Writing, 18(1), 7-24. 
Devlin, K. (2013). MOOCs and the myths of dropout rates and certification. Retrieved January 8 , 2014, from the Huffington Post Web site:. http://www.huffingtonpost.com/dr-keith-devlin/ moocs-and-the-myths-of-dr_b_2785808.html

Jarvis, H., \& Krashen, S. (2014). Is CALL obsolete? Language acquisition and language learning revisited in a Digital Age. TESL-EJ 17(4). Retrieved February 28, 2014, from the TESL-EJ Web site: http://www.tesl-ej.org/wordpress/issues/volume17/ej68/ej68a1/

Jordan, K. (2013). xMOOC? CMOOC? EDCMOOC - e-learning \& digital cultures. Retrieved April 19, 2014, from the MOOCMoocher Web site: http://moocmoocher.wordpress.com/2013/05/28/ xmooc-cmooc-edcmooc-e-learning-digital-cultures/

Kop, R. (2011). The challenges to Connectivist learning on open online networks: Learning experiences during a Massive Open Online Course. International Review of Research in Open and Distance Learning, 12.3.Retrieved April 15, 2013, from the National Research Canada Web site: http://nparc.cisti-icist.nrc-cnrc.gc.ca/npsi/ctrl?action=rtdoc\&an=18150443

Kulkarni, C., Wei, K.P., Le, H., Chia, D., Papadopoulos, K., Cheng, J., Koller, D., \& Klemmer, S.R. (2013). Peer and self assessment in massive online classes. ACM Transactions on ComputerHuman Interaction, 9(4), Article 39. Retrieved April 9, 2014, from the Stanford University $\mathrm{HCl}$ Group Web site: http://hci.stanford.edu/publications/2013/Kulkarni-peerassessment.pdf

Levy, M. (1997). CALL: Context and conceptualisation. Oxford: Oxford University Press.

Mackness, J. (2013). cMOOCs and xMOOCs - key differences. Retrieved April 9, 2013, from the Jenny Mackness Web site: http://jennymackness.wordpress.com/2013/10/22/cmoocs-and-xmoocskey-differences/

Marques, J. (2013). A short history of MOOCs and distance learning. Retrieved April 14, 2014, from the MOOC News and Reviews Web site: http://moocnewsandreviews.com/a-short-history-ofmoocs-and-distance-learning/

Marzio, M. (n.d.). Real English. http://www.real-english.com/

McGuire, R. (2013). Building a sense of community in MOOCs. Campus Technology, August, 31-33. Retrieved April 2, 2014, from the Campus Technology Web site: http://campustechnology.com/ Articles/2013/09/03/Building-a-Sense-of-Community-in-MO0Cs.aspx/

Piech, C., Chuang, D., Huang, J. Ng, A., Cheng, Z., Koller, D. (2013). Tuned models of peer assessment in MOOCs. Retrieved January 19, 2014 http://arxiv.org/pdf/1307.2579.pdf.

Richards, J.C. (2006). Communicative language teaching today. Cambridge, UK: Cambridge University Press.

Romeo, K. (2012). Language learning MOOCs? Retrieved April 1, 2014, from the Stanford University Web site: https://www.stanford.edu/group/ats/cgi-bin/hivetalkin/?p=3011

Schmidt, R., Boraie, D., \& Kassabgy, O. (1996). Foreign language motivation: Internal structure and external connections. In R. Oxford (Ed.), Language learning motivation: Pathways to the new century. (Technical Report \#11). (pp. 9-70). Honolulu: University of Hawai‘i, Second Language Teaching \& Curriculum Center.

Siemens, G. (2012). MOOCs are really a platform. Retrieved January 4, 2014 from the eLearnspace Web site: http://www.elearnspace.org/blog/2012/07/25/moocs-are-really-a-platform/

Smith, B. (1997). Virtual realia. The Internet TESL Journal, III(7). Retrieved April 20, 2014, from the iTESLJ Web site: http://iteslj.org/Articles/Smith-Realia

Stevens, V. (2013). What's with the MOOCs? TESL - EJ: The Electronic Journal for English as a Second Language, 16 (4), 1-14. Retrieved March 1, 2013, from the TESL-EJ Web site: http://www.tesl-ej. org/wordpress/issues/volume16/ej64/ej64int/

Tomkin, J.H., \& Charlevoix, D. (2014). Do professors matter? Using an a/b test to evaluate the impact of instructor involvement on MOOC student outcomes. Proceedings of the first ACM conference on Learning @ scale conference, 71-78. Retrieved April 19, 2014, from the ACM Digital LibraryWeb site: http://delivery.acm.org/10.1145/2570000/2566245/p71-tomkin.pdf 
WGBH Boston (1987). French in action. Retrieved April 14, 2014, from the WGBH Web site: http:// main.wgbh.org/wgbh/learn/instructional/french.html

Wukman, A. (2012). Coursera battered with accusations of plagiarism and high drop-out rates.

Online Colleges. Retrieved January 4, 2014, from the Online Colleges Web site: http://www. onlinecolleges.net/2012/08/22/coursera-battered-with-accusations-of-plagiarism-and-highdrop-out-rates/ 


\section{Appendix}

\section{Rubric for Self-assessment of Writing}

Select the criteria you feel best represents this submission in each category.

\section{Thesis}

0 points: I still don't understand how to write a good thesis.

1 point: I'm not confident about my thesis at all, but I tried.

2 points: I think my thesis is okay, but I am concerned I don't have a strong argument.

3 points: I wrote a strong thesis I can identify and a main idea that others might have different opinions about.

\section{Content}

0 points: I didn't know how to support my ideas.

1 point: I wasn't always sure how to supply support for ideas, but I think some of it is okay. I need to review that course material again.

2 points: I support most of my ideas, but it seems some of them might just be personal opinion.

3 points: I support my ideas with clear facts and ideas in each paragraph. I can identify my examples easily.

\section{Organization}

0 points: I wasn't sure how to organize it. I don't think it's organized at all.

1 point: I'm worried that my ideas might seem random and unconnected to each other. I need to work on this more, but I tried.

2 points: I tried to organize my ideas logically. I used signposts and transitions, but there are probably some problems.

3 points: I outlined my work and I'm confident that it has a clear and logical organization, using correct transitions.

\section{Vocabulary}

0 points: I'm not sure how to do better on vocabulary.

1 point: I didn't take the time to look up my vocabulary, and there might be a lot of misused words.

2 points: I wasn't sure about a few words, but overall, I think it's accurate.

3 points: I am confident that my vocabulary is effective and accurate. 


\section{Grammar and Punctuation}

0 points: My grammar needs a lot of work!

1 point: I am still working on my grammar, but I made efforts to do well. There are probably a lot of mistakes.

2 points: I think I did a good job on grammar and punctuation, but there might be more than a few mistakes.

3 points: I used a grammar checker online or in my word-processing system, and asked someone else to read it. I'm sure it's nearly perfect in grammar and punctuation. 